\title{
Vibration parameters measuring system of rotate mechanical based on MEMS sensor
}

\author{
Jun Chen*, and Tao Zhou \\ Luoyang Normal University, Physics and Electronic Information Department, 471934 Luoyang, \\ China
}

\begin{abstract}
In this work, a novel vibration parameter measure instrument of rotate machine using a accelerometer is proposed. The ADXL203 micro accelerometer is used as the sensor of the instrument based on the MEMS (Micro Electro Mechanical System) technology. The mathematical modeling of the vibration is finished by the simple harmonic vibration theory. The noise of the system is disposed by the differential noise reduction circuit. The experiments such as differential noise reduction, frequency and vibration amplitude measurement are finished on the platform. The results indicate that the instrument is useful and effective.
\end{abstract}

\section{Introduction}

Rotation machineries are the essential parts of the modern industry. During the long term work, various kinds of vibrations are arise inevitably due to the change of the work environment, parts of fatigue, key component loose and so on $[1,2]$. The vibration can induce a series of resonances on the other components. If these vibrations are not monitored persistently, the fracture crack damage will happen at the connectors of pumps, the instruments on line, the welded joints and all the other parts that we defined as the vulnerable spots [3]. So the vibrations from the rotation machineries under steady and transient conditions must be analyzed in real-time and uninterruptedly monitored in order to muster the characteristics. Also the diagnosis and prediction methods should be established. The vibration parameters measure system are designed to measure the important vibration parameters under the working condition. Based on the parameters, the accident judgments should be passed immediately, which will protect the machineries and the production equipment. This paper realized a vibration measuring instrument based on the MEMS sensor, by which the vibration amplitude and frequency can be measured online in real-time.

\section{System overview}

The measure system includes two parts: the vibration detector, and the principle computer analyze system. The diagram of the system is shown in figure 1. From the technics review it is known that the MEMS accelerometer is best for the vibration measurement due to its

\footnotetext{
*Corresponding author: chenjun471000@163.com
} 
light weight, tiny body, low power consumption, multi-function, three measuring axises and low noise. The rectify circuit is designed to amplify the signal from the sensor. These processes are all for the calculation in the controller. Sometimes, the working environment is not good enough to dispose a computer, then the controller undertakes the calculating tasks of accelerating velocity, speed and amplitude. So it is necessary to choose a controller with high performance to finish the tasks. The distributed wireless vibration monitoring system based on the wireless sensor can realize the long distance signal transmission [4].

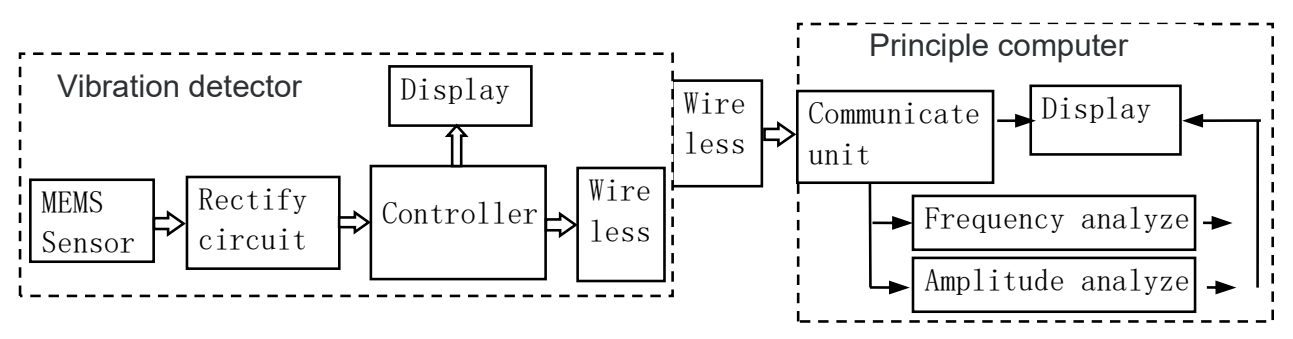

Fig.1. System diagram.

The software in the principle computer is developed form the virtual instrument technology from NI. The software is of strong ability in data calculating, analyzing, display, and communication. Based on this, the developing period and difficulty are reduced a lot.

\section{Simple harmonic vibration method}

The periodic vibration peak value of the rotation machines is rolling in some range. The maximum vibration peak value is the amplitude that need to be monitored. This kind of vibration can be described as the simple harmonic vibration using spring oscillator. First, the maximum accelerated velocity should be measured at the peak position of vibration. Second, the frequency can be calculated. Last, the amplitude result is got by the function related to simple harmonic vibration theory.

The simple harmonic vibration theory is shown in figure 2. The maximum vibration accelerated velocity is $a_{\max }$, and the minimum vibration accelerated velocity is $a_{\min }$. The mass of spring oscillator is $m$, and the spring stiffness factor is $k$. Here $m$ and $k$ are all constants. Assume the amplitude of spring oscillator is $x$. From the theory of Newtonian mechanics, the equation of spring oscillator motion is equation (1):

$$
F=\mathrm{k} x=\mathrm{m} a=\mathrm{m} \ddot{x}
$$

The differential equation is (2):

$$
\mathrm{k} x=\mathrm{m} \ddot{x}
$$

From (2), the solution is equation (3):

$$
x=\mathrm{A} \cos \left(\sqrt{\frac{\mathrm{k}}{\mathrm{m}}} t+\mathrm{t}_{0}\right)
$$

Which $A$ is the maximum vibration peak value, $t_{0}$ is the time constant.

So the vibration period is equation (4):

$$
T=2 \pi \sqrt{\frac{\mathrm{k}}{\mathrm{m}}}
$$


Then equation (5):

$$
\mathrm{k}=\mathrm{m}\left(\frac{T}{2 \pi}\right)^{2}
$$

Take (5) into (2) is equation (6):

$$
\left(\frac{T}{2 \pi}\right)^{2} x=\ddot{x}
$$

Mark the $\ddot{x}$ as $a$, then the amplitude $x$ is equation (7):

$$
x=\frac{4 \pi^{2} a}{T^{2}}
$$

From figure 2, and (7), when the $a$ is $a_{\max }$, the amplitude $x$ is the max value $+x$, which is the vibration amplitude that needs to be measured. According to the FFT and "Bubble Method", the vibration period $T$ and $a_{\max }$ can be got. At last, using (7), the max amplitude $+x$ will be obtained.

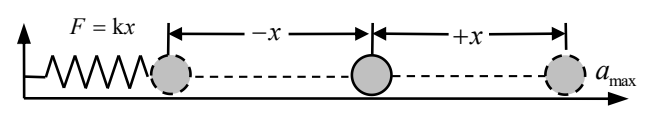

Fig. 2. Simple harmonic vibration.

\section{Acceleration calculation}

The acceleration value from the sensor contained an offset value, not the real value. So the accelerometer contains an bias acceleration between $+1 \mathrm{~g} \sim-1 \mathrm{~g}$. While the vibration measure instrument is working, this bias will be added in the real vibration acceleration which can disturb the final displacement value, so it must be removed.

From the software, many vibration periods are collected. Among these periods, the max and min accelerations are picked out by the bubble sort algorithm and the average acceleration value is calculated by the max and min acceleration. This average acceleration value is the balance position of the displacement accumulation algorithm [5]. The one quarter period which include the max value is picked out and after each value (sample point) of the period minuses the average acceleration value one by one. Then the DC voltage bias between $+1 \mathrm{~g} \sim-1 \mathrm{~g}$ is removed and left the real acceleration value.

\section{Hardware of the accelerometer}

\subsection{Circuit of the sensor}

In order to reduce the coupling effect and the noise from the power, the output voltage signal is filtered by the $0.1 \mu \mathrm{F}$ capacitance $\mathrm{C}_{\mathrm{DC}}$, which method is widely used in circuit design. But in some situations, especially when the noise frequency is about $140 \mathrm{KHz}$, the ADXL203 output maybe disturbed by the power noise (figure 3). So a $100 \Omega$ (even smaller) resistance is added in the power wire. Furthermore, a large capacitance $(1 \mu \mathrm{F} \sim 22 \mu \mathrm{F})$ can be parallelly connected at $\mathrm{C}_{\mathrm{DC}}$. 

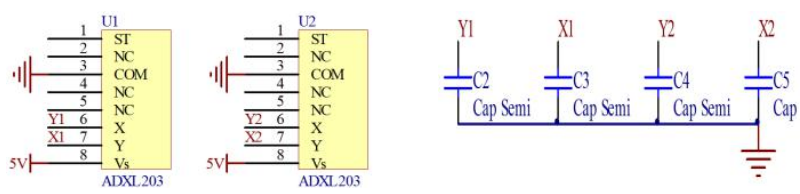

Fig. 3. ADXL203 schematic.

\subsection{Noise reduction circuit}

The noise reduction circuit is designed to increase the SNR. The double sensors orthogonal difference method is utilized. With the axis direction of the sensor, the double orthogonal X axises are used as the measure axis. One is the same direction with vibration, named measurement axis. The other axis is the reference axis. In this way, the output signals from the two orthogonal axises are subtracted each other in the circuit, so the noise is dispelled. The circuit is in figure 4 .

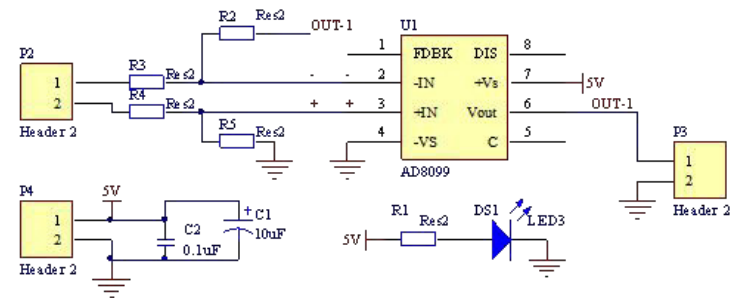

Fig. 4. Noise reduction circuit.

\section{Software design}

\subsection{VISA design}

VISA is the standard I/O API of instrument programming. There are many applications: the GBIP controlling, serial port, USB, internet, PXI or VXI. The drive programs can be called by every kinds of instruments. The users do not need to study the communication protocol of each kind of instrument. The VISA is independent from the operation system and program environment. In another word, the same API can be used in any instrument, operation system, and program langrage. Before we use the VISA, make sure that the control method is correct. The most used functions based on the message are: VISA read, VISA write, VISA trig, VISA clear, and VISA read STB. These functions are in the figure5.

Configurate the port is the first step to use the VISA port. The main parameters are: number of the port, bund rate, check bit, and data bits. The bund rate is set as 115200 . The port number will be selected automatically by the principle computer after the port connected. The data is 8 bits default, and without check bit. They are shown in figure 6 .

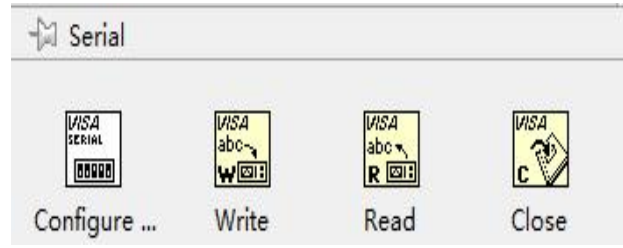

Fig. 5. Serial port configuration controls.

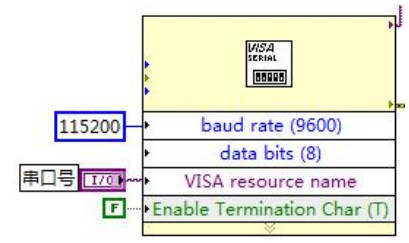

Fig. 6. Configurate the port.

\subsection{FFT algorithm}


By the circuits in 4.2, the signal has reduced the noises. The vibration frequency will be calculated by using the FFT algorithm. With the frequency, the amplitude and speed can be obtained by the theory mentioned in chapter 2 . The program is in figure 7 .

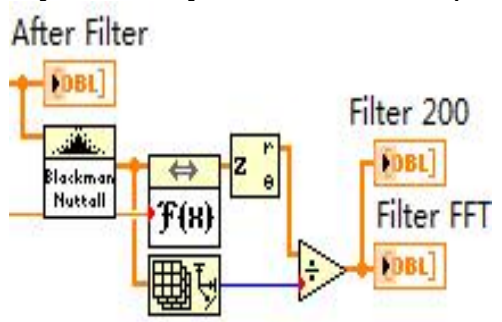

Fig. 7. FFT program.

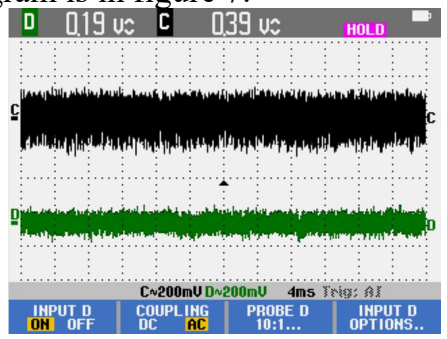

Fig. 8. Signal wave form from difference circuit.

Before FFT, the Blackman window control is added, in order to smooth the spectrum leakage which existed in the beginning and end of the data storage. After the Blackman window, the real part of the FFT data is picked out by the control of " $\mathrm{Z}_{\theta}^{\mathrm{r}}$ ". These data are the frequency data of the vibration. Each data correspond to a frequency from $5 \mathrm{~Hz}-8192 \mathrm{~Hz}$. With the help of band-pass filter, the vibration amplitude above $1000 \mathrm{~Hz}$ tend to zero little by little. After the FFT of 8192 points, the main frequencies will be read out in the front panel. At last, the maximum frequency data will be obtained, which can be used to calculate the amplitude.

\section{Experiment and data analysis}

\subsection{Differential filter}

Figure 8 is the signal output waveform with the length $4 \mathrm{~ms}$. The unit of the oscilloscope is $200 \mathrm{mv}$. The peak-peak value of the original signal is $0.39 \mathrm{~V}$ in the measurement axis before the signal is transmitted into the differential circuit board. After the signal is transmitted out from the circuit board, the peak-peak value of the signal is $0.19 \mathrm{~V}$, decrease about $0.2 \mathrm{~V}$, about $51.3 \%$ compered with the original signal. This indicated that the differential circuit board realized good results in noise reduction.

In the real piping mechanical engineering, the water follow speed is controlled by the three-phase magnetic pump. The speed waveforms of different speed are shown in figure 9 . The channel $\mathrm{C}$ of the oscilloscope indicates the original signal, channel $\mathrm{D}$ indicates the signal from the differential circuit. In the continuous vibration experiments, two figures were obtained. The sample length of figure (a) is $100 \mu$ s. The peak-peak value of the original noise signal is $0.47 \mathrm{~V}$. After the differential circuit operation, the peak-peak value of the noise is reduced to $0.22 \mathrm{~V}$. The peak-peak value of the original noise signal is $0.46 \mathrm{~V}$. After the differential circuit operation, the peak-peak value of the noise is reduced to $0.18 \mathrm{~V}$.

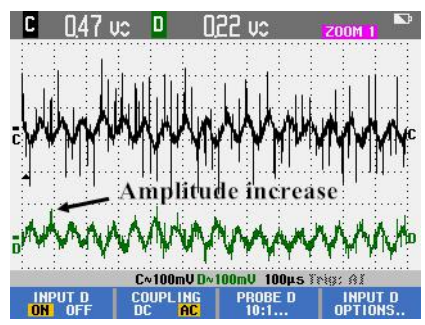

(a) $100 \mu$ s length wave

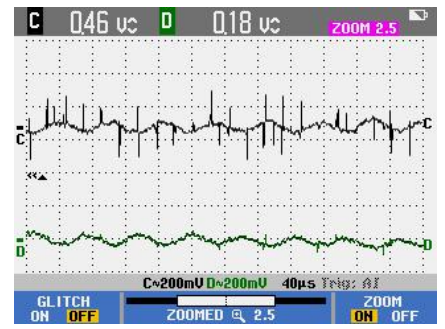

(b) $40 \mu$ s length wave

Fig. 9. Pipe vibration waveform. 
The signals from two channels are subtracted due to the differentiate operation. The one is from measuring channel, the other is from reference channel. Although the differences between the two chips can be neglected, the sources of the noises are not all the same. The phase positions of the two signals at the same time maybe not the same. If the phase positions are opposite, the amplitude maybe increase after the differential circuit operation. This phenomenon appeared in channel $\mathrm{D}$ in figure 9 (a). But in figure 9 (b), this phenomenon is weak. In one word, after the band-pass filtering, these peak noises will not influence the signal process that followed behind.

\subsection{Vibration frequency test}

On the motor vibration experiment rig, the motor speed is adjusted from $50 \mathrm{~Hz}$ to $40 \mathrm{~Hz}$. According to the FFT on the principal computer, the spectrograms are drawn in the figure 10 and figure 11. From the spectrograms at $50 \mathrm{~Hz}$ and $40 \mathrm{~Hz}$, the synchronous speed of the magnetic could not influence the motor vibration. The vibration results are from the vibration fundamental wave on the motor shaft rotation. At synchronous frequency $50 \mathrm{~Hz}$, the motor rotate frequency is about $47 \mathrm{~Hz}$. At synchronous frequency $40 \mathrm{~Hz}$, the motor rotate frequency is about $38 \mathrm{~Hz}$. The two rotate frequencies keep perfect accordance with the speeds. The results indicated that the frequency measurement is effective.

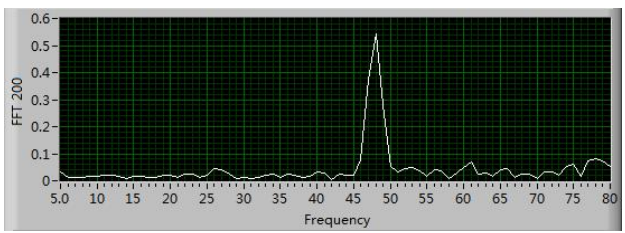

Fig. 10. $50 \mathrm{~Hz}$ water pump vibration FFT.

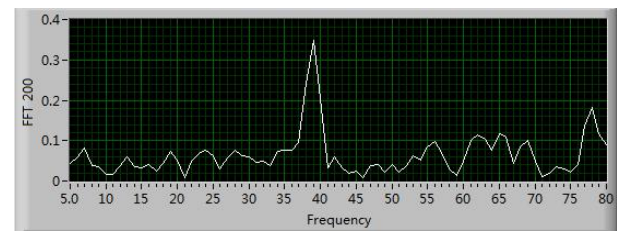

Fig. 11. $40 \mathrm{~Hz}$ water pump vibration FFT.

\subsection{Vibration amplitude test}

The uniaxial piezoelectric vibration measuring system from NI is used as the reference standard sensor to measure the vibration amplitude. The pipe vibration amplitude is adjusted according to the change of the rotating speed of the water pump. The amplitude algorithm is tested and corrected with continuous comparison to the results from the reference standard sensor. From $1 \mu \mathrm{m}$ to $10 \mu \mathrm{m}$, and increased $1 \mu \mathrm{m}$ each time. After being tested many times, each result is amended the amplitude error. The accurate experiment results are shown in figure 12.

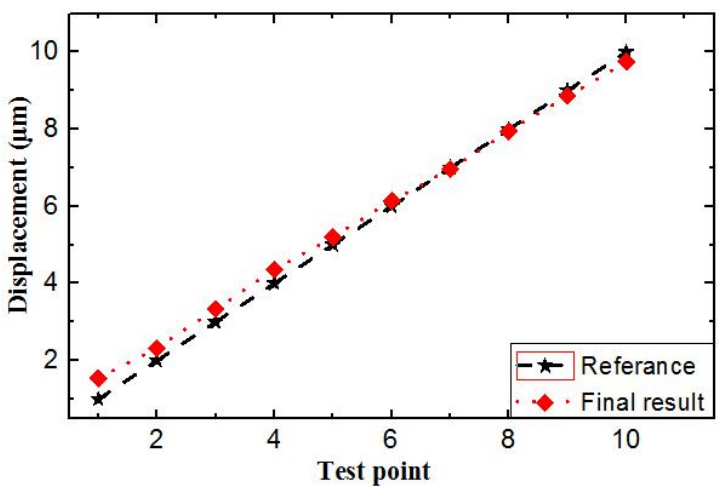

Fig. 12. Comparative curve of vibration amplitudes. 
Compared with the reference amplitudes, the maximum error from the measure system is $0.38 \mu \mathrm{m}$, average error is $0.22 \mu \mathrm{m}$. The maximum error in the slope of matching curve is 0.0024 . The intercept error is 0.0923 . These results illustrated that the amplitude measurement is linearizing and accurate.

\section{Conclusion}

Aiming at the characteristic of the rotating machinery vibration, a novel vibration parameter measuring instrument of rotate machine using a accelerometer was proposed based on the MEMS technic. The characteristic of the rotating machinery vibration were analyzed. In order to reduce the noise and increase the SNR, the differential noise reduction circuit was designed and tested. By using the virtual instrument technology, the software was compiled. The data communication function is constructed by the VISA. At last, the experiment results showed that this measuring equipment could reduce the noise, calculating the frequency, and vibration magnitude.

\section{Acknowledgments}

The authors wish to acknowledge the financial support of Science and technology key project in Henan province (Grant No.182102210430) and (Grant No.182102210435), Application Fund Project of Luoyang Normal University (Grant No.4320024).

\section{References}

1. Liu Li, Zhang xiaobin, Qiu Limin, Liu Li. Vibration reason analysis and vibration reduction methods of Piping system in thermal power plant. Cryogenic Engineering, 04, 50 (2016)

2. ZHAO Xinghai, ZHAI Song, PENG Longfei, XING Jingwei, XIN Guohua. Vibration reason analysis and vibration reduction methods of Piping system in thermal power plant [J]. Boiler Technology, 1 (44), 67 (2003)

3. YUAN Wei, ZHAO Jie, LI Feng, LIU Zhengtong. Mechanism of Vibration Occurred in Compressor Pipeline and Summary of Study in Techniques for Vibration Reduction. Process Equipment \& Piping, 53(2), 36 (2016)

4. Tang Baoping, Huang Qingqing, Deng Lei, Liu Ziran . Research progress and challenges of wireless of sensor networks for machinery equipment condition monitoring. Journal of Vibration, Measurement \& Diagnosis, 34(1), 1 (2014)

5. CHEN Jun, ZHOU Tao. A new kind of vibration frequency measure system of grinder. Computer measurement and control, 10 (23), 3350 (2015) 